\title{
Accelerator based neutron source for neutron capture therapy
}

B. Bayanov, Yu. Belchenko, V. Belov, V. Davydenko, A. Donin, A. Dranichnikov, A. Ivanov, I. Kandaurov, G. Kraynov, A. Krivenko, A. Kudryavtsev, N. Kuksanov, R. Salimov, V. Savkin, V. Shirokov, I. Sorokin, S. Taskaev, M. Tiunov

Budker Institute of Nuclear Physics, Novosibirsk, Russia

The Budker Institute of Nuclear Physics (Novosibirsk) and the Institute of Physics and Power Engineering (Obninsk) have proposed an accelerator based neutron source for neutron capture and fast neutron therapy for hospital. Innovative approach is based upon vacuum insulation tandem accelerator (VITA) and near threshold ${ }^{7} \mathrm{Li}(p, n)^{7} \mathrm{Be}$ neutron generation. Pilot accelerator based neutron source for neutron capture therapy is under construction now at the Budker Institute of Nuclear Physics, Novosibirsk, Russia. In the present report, the pilot facility design is presented and discussed. Design features of facility components are discussed. Results of experiments and simulations are presented. Complete experimental tests are planned by the end of the year 2005.

\section{Introduction}

The Budker Institute of Nuclear Physics, and the Institute of Physics and Power Engineering, Obninsk, have proposed an accelerator based neutron source for neutron capture and fast neutron therapy for hospital (Bayanov, 1998). Innovative approach is based upon vacuum insulation tandem accelerator (VITA) and near threshold ${ }^{7} \operatorname{Li}(\mathrm{p}, \mathrm{n}){ }^{7} \mathrm{Be}$ neutron generation. Pilot facility is under construction now at the Budker Institute of Nuclear Physics.

In the present report, the pilot facility design is presented and discussed. Design features of facility components are discussed. Current status of project realization, results of experiments and simulations are presented.

\section{Accelerator based neutron source conception}

Pilot variant of neutron therapy facility is shown Fig. 1. Negative hydrogen ion beam is injected into electrostatic vacuum insulation tandem accelerator (VITA). After charge-exchange of negative hydrogen ion into proton inside the charge-exchange tube in the center of high-voltage electrode, the proton beam is formed at the outlet of the tandem. It is accelerated up to double voltage of high-voltage electrode. Neutron generation is proposed to be carried out by protons bombarding a lithium target using ${ }^{7} \mathrm{Li}(p, n){ }^{7} \mathrm{Be}$ threshold reaction.

In ordinary mode, at proton energy of $2.5 \mathrm{MeV}$, the neutron source produces neutron beam with maximum energy of $790 \mathrm{keV}$ appropriate directly for fast neutron therapy and for neutroncapture therapy after moderation.

The most efficient operating mode of facility is at proton energy of $1.915 \mathrm{MeV}$ that is $34 \mathrm{keV}$ higher than the threshold of the ${ }^{7} \mathrm{Li}(p, n)^{7} \mathrm{Be}$ reaction. In this mode, neutron beam is generated kinematically collimated in forward direction and its average energy of $30 \mathrm{keV}$, is directly applicable for boron neutron-capture therapy. 


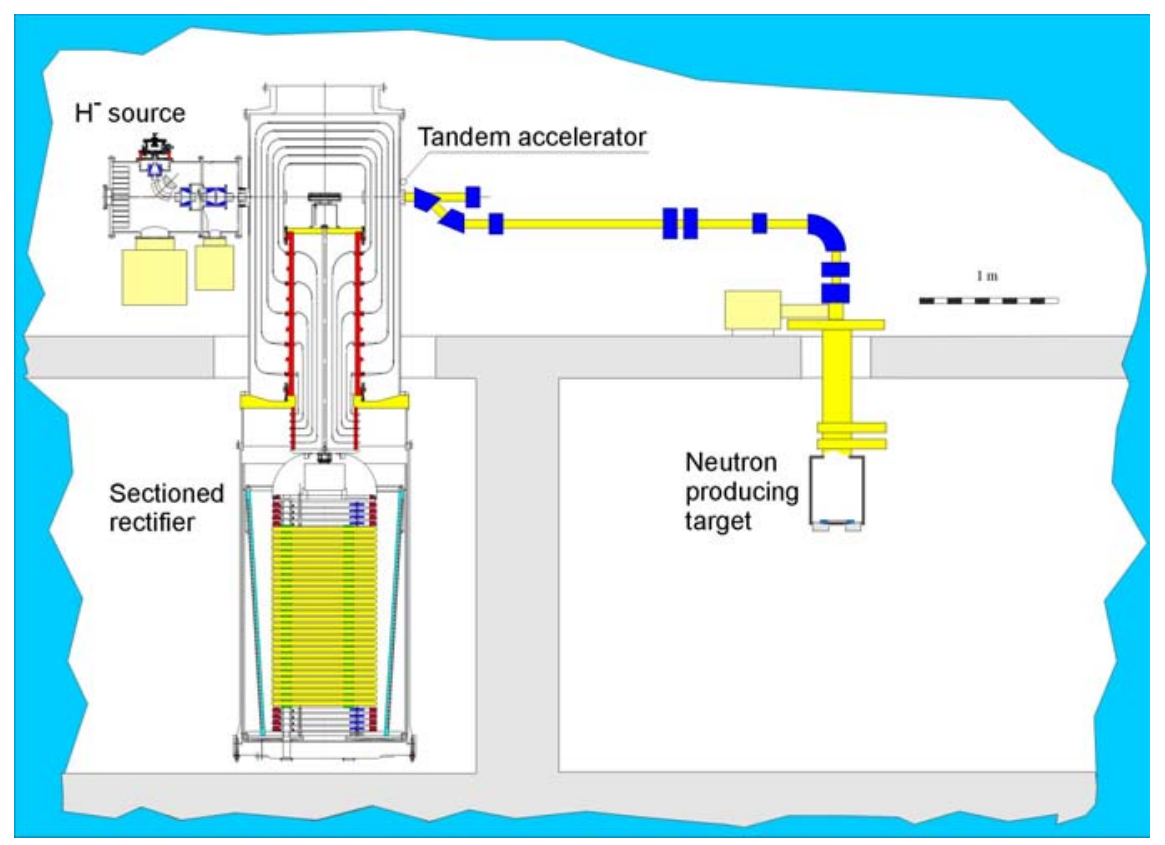

Fig. 1. Pilot variant of accelerated based neutron source for NCT.

\section{Discussion}

Surface-plasma negative ion source with Penning geometry of electrodes (Belchenko, 2002) is to be used for obtaining a dc $25 \mathrm{keV} 10 \mathrm{~mA}$ beam of hydrogen negative ions. Now, a dc $\mathrm{H}^{-}$ion beam of $5 \mathrm{~mA}$ has been obtained and investigated on the prototype of negative ion source with Penning geometry of electrodes. The value measured for the normalized emittance of $0.3 \pi \mathrm{mm}$ mrad meets the requirements. Negative ion source with Penning geometry electrodes and power supply is being fabricated (Belchenko, 2004).

Beam transporting to the accelerator is fulfilled with two magnetic lenses movable lengthwise. Coordinated input of the beam into the accelerator will be realized in one of the two following ways (Derevyankin, 2002): "strong" scheme with the use of strong magnetic lens and beam over focusing at the entrance to accelerator, and "weak" introduction without beam over focusing with increased first gap and more smooth increase of electric field intensity in accelerator. Since both of the schemes have advantages and disadvantages and both of them have only slight difference in the ion-optical path design, the recommended solution is to provide the possibility to check both "weak" and "strong" beam focusing experimentally. Magnetic lens with outer water cooling was designed and manufactured. Additional experiments on pulsed beam transporting are planned to be fulfilled in the nearest future (Davydenko, 2004).

In the accelerator, the beam is accelerated in the electric field of $33 \mathrm{kV} \mathrm{cm}^{-1}$. Five intermediate and one high-voltage electrode $600 \mathrm{~mm}$ in diameter are placed inside a vacuum tank $1400 \mathrm{~mm}$ in diameter. Inside the high-voltage electrode, stripping gas target is placed (Derevyankin, 2001), made as a tube $400 \mathrm{~mm}$ length $10 \mathrm{~mm}$ in diameter. To decrease gas flow in accelerating channel, gas recirculation with turbo-molecular pump inside the high-voltage electrode is provided (Davydenko, 2004). Vacuum volume is to be pumped out through the profiled covers of intermediate electrodes with cryogenic pump placed on top. The vacuum chamber for the accelerator has been fabricated.

Potential is feed to the high-voltage electrode through insulator from $40 \mathrm{~kW} 1.25 \mathrm{MV}$ sectioned rectifier of ELV-type industrial electron accelerator (Salimov, 2000). The sectioned rectifier is mounted and tested at $1.4 \mathrm{MV}$ voltage for continuous steaming operation. Voltage stabilization system was proposed (Veis, 2002.). Heat carrier input for forced cooling of stripping 
target and recirculation turbo-molecular pump and pump feeding are provided in the feedthrough insulator design. The feedthrough insulator is being manufactured now.

Neutrons are generated by protons bombarding the target covered with thin solid lithium layer. The target is described in detail in (Bayanov, 2004; Bayanov, 2005).

\section{Conclusion}

We plan to manufacture the main components of the facility up to the end of 2004 and complete experimental tests by the end of 2005 .

\section{References}

Bayanov, B. et al, 1998. Accelerator based neutron source for the neutron-capture and fast neutron therapy at hospital, Nucl. Instr. and Meth. in Phys. Res. A 413, pp. 397-426.

Bayanov, B. et al, 2004. Optimization of lithium target for epithermal neutrons generation. Intern. Symposium on NCT, July 7-9, Novosibirsk, Russia.

Bayanov, B. et al, 2005. Lithium neutron producing target for BINP accelerator based neutron source. Applied Radiation and Isotopes.

Belchenko, Y., Grigoryev, E., 2002. Surface-plasma negative ion source for the medicine accelerator. Rev. Sci. Instrum. 73, p. 939.

Belchenko, Y., 2004, Intern. Symposium on NCT, July 7-9, Novosibirsk, Russia.

Davydenko V., Krivenko A., 2004. Stripping target for a $10 \mathrm{~mA}, 1 \mathrm{MeV}$ negative-hydrogen ion beam. Intern. Symposium on NCT, July 7-9, Novosibirsk, Russia.

Davydenko V., et. al. Test experiments for ion beam injection at the prototype of electrostatic tandem accelerator. Intern. Symposium on NCT, July 7-9, Novosibirsk, Russia.

Derevyankin, G. et al, 2001. Charge-exchange target for $40 \mathrm{~mA} 2 \mathrm{MeV}$ tandem accelerator. Preprint BINP 2001-23. Novosibirsk.

Derevyankin, G. et al, 2002. The ion-optical channel of $2.5 \mathrm{MeV} 10 \mathrm{~mA}$ tandem accelerator. Preprint BINP 2002-24. Novosibirsk.

Salimov, R. et al., 2000. Radiation Physics and Chemistry 57, pp. 661-665.

Veis, M. et al., 2002. Stabilization of accelerating voltage in HV-tandem accelerator for neutron capture therapy. Preprint BINP 2002-24. Novosibirsk. 